Pacific Journal of Mathematics

BANACH-BUCK MEASURE, DENSITY, AND UNIFORM
DISTRIBUTION IN RINGS OF ALGEBRAIC INTEGERS 


\title{
BANACH-BUCK MEASURE, DENSITY, AND UNIFORM DISTRIBUTION IN RINGS OF ALGEBRAIC INTEGERS
}

\author{
SiU Kwong Lo and H. Niederreiter
}

The theory of uniform distribution of sequences of algebraic integers in a fixed algebraic number field $K$, as initiated by Kuipers, Niederreiter, and Shiue, is developed from a measuretheoretic viewpoint. After establishing some general facts in $\S 2$, in particular, the analogy between uniform distribution of sequences of algebraic integers in $K$ and of sequences of lattice points, a method of enumerating all algebraic integers in $K$ into a uniformly distributed sequence is discussed in $\S 3$. This enumeration method is useful for the construction of other uniformly distributed sequences as well and plays a role in the density theory. In $\S 4$, a so-called Banach-Buck measure is defined on the ring of all algebraic integers in $K$. Various relations between this measure and the property of uniform distribution are exhibited. Based on Buck's general concept of density, the notions of relative density and of density of sets of algebraic integers in $K$ are introduced in the final section. Connections among the concepts of uniform distribution, measurability, and relative density of sequences of algebraic integers in $K$ are established.

1. Introduction. The definition of uniform distribution of sequences of algebraic integers in a fixed algebraic number field $K$ was introduced by Kuipers, Niederreiter, and Shiue [5]. In the present paper, we shall develop the theory from a measure-theoretic viewpoint.

After establishing some general facts in $\S 2$, in particular, the analogy between uniform distribution of sequences of algebraic integers in $K$ and of sequences of lattice points, we discuss in $\S 3$ a method of enumerating all algebraic integers in $K$ into a uniformly distributed sequence. This enumeration method is useful for the construction of other uniformly distributed sequences and plays a role in the density theory. In $\S 4$, we define a so-called Banach-Buck measure on the ring of all algebraic integers in $K$. Various relations between this measure and the property of uniform distribution are exhibited. Based on Buck's general concept of density, we introduce in the final section the ideas of relative density and of density of sets of algebraic integers in $K$. We establish connections among the concepts of uniform distribution, measurability, and relative density of sequences of algebraic integers in $K$. A variety of interesting problems emerge in this study. 
2. Generalities. Let $K$ be a given algebraic number field of degree $[K: Q]=k$ over the rationals, and let $O$ be the ring of all algebraic integers in $K$. Let $I \subset O$ be a nontrivial integral ideal with norm $\mathscr{N} I$. If $\mathscr{A}=\left(\alpha_{n}\right), n=1,2, \cdots$, is a sequence of elements in $O$, then $A(N, \alpha+I, \mathscr{A})$ will denote the number of $n, 1 \leqq n \leqq N$, such that $\alpha_{n} \equiv \alpha(\bmod I)$. The following two definitions can be found in [5].

DEFINITION 2.1. Let $I \subset O$ be a nontrivial integral ideal. Then the sequence $\mathscr{A}$ is uniformly distributed modulo $I$ (u.d. $\bmod I$ ) if

$$
\lim _{N \rightarrow \infty} \frac{A(N, \alpha+I, \mathscr{A})}{N}=\frac{1}{\mathscr{N} I}
$$

for every $\operatorname{coset} \alpha+I$ of $I$.

DEFINITION 2.2. The sequence $\mathscr{A}$ is uniformly distributed in $O$ (u.d. in $O$ ) if $\mathscr{A}$ is u.d. $\bmod I$ for every nontrivial integral ideal $I \subset O$.

LEMMA 2.3. Let $J \subseteq I$ be nontrivial integral ideals, and let $\mathscr{A}$ be a sequence of algebraic integers in $O$. Then, if $\mathscr{A}$ is u.d. $\bmod J$, it is also u.d. $\bmod I$.

Proof. This follows immediately from the identity

$$
A(N, \alpha+I, \mathscr{A})=\Sigma A(N, \beta+J, \mathscr{A}),
$$

where the sum is taken over all distinct $\operatorname{cosets} \beta+J$ with $\beta \equiv \alpha(\bmod I)$.

THEOREM 2.4. The sequence $\mathscr{A}$ of algebraic integers in $O$ is u.d. in $O$ if and only if $\mathscr{A}$ is u.d. modulo the principal ideal mO for every rational integer $m \geqq 2$.

Proof. The necessity is trivial. To prove sufficiency, we choose a nontrivial integral ideal $I \subset O$. Since $O / I$ is an additive group of order $\mathscr{N} I$, we have the coset identity $(\mathscr{N} I)(1+I)=I$, and so $(\mathscr{N} I) O \subseteq I$. The rest follows from Lemma 2.3.

If an integral basis $W=\left\{\omega_{1}, \cdots, \omega_{k}\right\}$ of $K$ over $\boldsymbol{Q}$ is chosen, then for every $\alpha \in O$ we have $\alpha=\sum_{i=1}^{k} x_{i} \omega_{i}$ with $x_{i} \in Z$ for $1 \leqq i \leqq k$. The identification of $\alpha$ with the lattice point $\left(x_{1}, \cdots, x_{k}\right)$ provides a group isomorphism between $O$ and $\boldsymbol{Z}^{k}$. Thus, all the subsequent results that depend only on the set-theoretic or additive structure of $O$ have an analogue in $Z^{k}$, and vice versa. In the sequel, we shall 
sometimes identify a sequence in $O$ with one in $\boldsymbol{Z}^{k}$, and vice versa. An equivalent form of the following definition can be found in [6].

DEFINITION 2.5. Let $\underline{X}=\left(\underline{x}_{n}\right), n=1,2, \cdots$, be a sequence of lattice points in $\boldsymbol{Z}^{k}$. Then $\underline{X}$ is called u.d. $\bmod m \boldsymbol{Z} \oplus \cdots \oplus m \boldsymbol{Z}$, $m \geqq 2$ a rational integer, if

$$
\lim _{N \rightarrow \infty} \frac{1}{N} A\left(N,\left(j_{1}, \cdots, j_{k}\right)+(m \boldsymbol{Z} \oplus \cdots \oplus m \boldsymbol{Z}), \underline{X}\right)=\frac{1}{m^{k}}
$$

for every $\left(j_{1}, \cdots, j_{k}\right) \in \boldsymbol{Z}^{k}$, the counting function having the obvious meaning. Furthermore, if $\underline{X}$ is u.d. $\bmod m \boldsymbol{Z} \oplus \cdots \oplus m \boldsymbol{Z}$ for every $m \geqq 2$, then $\underline{X}$ is called u.d. in $Z^{k}$.

If one identifies the integral ideal $m O$ of $O$ with the subgroup $m \boldsymbol{Z} \oplus \cdots \oplus m \boldsymbol{Z}$ of $\boldsymbol{Z}^{k}$, then in view of Theorem 2.4 and Definition 2.5 , the uniform distribution of a sequence in $O$ is equivalent to the uniform distribution of the corresponding sequence in $Z^{k}$.

We shall write $\exp (t)=e^{2 \pi i t}$ for any real number $t$. Furthermore, if $\underline{a}=\left(a_{1}, \cdots, a_{k}\right)$ and $\underline{b}=\left(b_{1}, \cdots, b_{k}\right)$ are two vectors of the euclidean space $\boldsymbol{R}^{k}$, then $\underline{a} \cdot \underline{b}=\sum_{i=1}^{k} a_{i} b_{i}$ will denote their standard inner product. The following is well-known (see [10] for the case $k=1$ and [6] for the general case).

THEOREM 2.6 (Weyl criterion). A sequence $\underline{X}=\left(\underline{x}_{n}\right), n=1,2, \cdots$, in $\boldsymbol{Z}^{k}$ is u.d. $\bmod m \boldsymbol{Z} \oplus \cdots \oplus m \boldsymbol{Z}$ if and only if

$$
\lim _{N \rightarrow \infty} \frac{1}{N} \sum_{n=1}^{N} \exp \left(\left(\frac{j_{1}}{m}, \cdots, \frac{j_{k}}{m}\right) \cdot \underline{x}_{n}\right)=0
$$

for all those $\left(j_{1}, \cdots, j_{k}\right) \in Z^{k}$ for which not all of the coordinates are divisible by $m$.

3. The cube method. We describe a method of obtaining a u.d. sequence in $Z^{k}$ out of $k$ sequences that are u.d. in $\boldsymbol{Z}$. Suppose we are given $k$ sequences $X_{1}, \cdots, X_{k}$ of rational integers with $X_{i}=\left(x_{n i}\right)$, $n=1,2, \cdots$, for $1 \leqq i \leqq k$. Then the lattice point $\left(x_{n_{1}, 1}, \cdots, x_{n_{k}, k}\right)$ is said to lie in the $m$ th cube if $\max _{j=1, \ldots, k} n_{j}=m$. We now enumerate all elements of the form $\left(x_{n_{1}, 1}, \cdots, x_{n_{k}, k}\right)$ by starting with the element in the first cube, then enumerating all the elements in the second cube in an arbitrary order, then all the elements in the third cube in an arbitrary order, and so on. Then a sequence in $Z^{k}$ resulting from this construction is called a sequence arising from $X_{1}, \cdots, X_{k}$ by the cube method.

THEOREM 3.1. A sequence $\underline{Y}=\left(\underline{y}_{n}\right), n=1,2, \cdots$, arising from 
the sequences $X_{1}, \cdots, X_{k}$ by the cube method is u.d. in $Z^{k}$ if and only if $X_{i}$ is u.d. in $\boldsymbol{Z}$ for $i=1, \cdots, k$.

Proof. Suppose first that each $X_{i}=\left(x_{n i}\right), n=1,2, \cdots$, is u.d. in $\boldsymbol{Z}$. Choose a rational integer $m \geqq 2$ and $\left(j_{1}, \cdots, j_{k}\right) \in Z^{k}$, not all of the $j_{i}$ being divisible by $m$. Then,

$$
\begin{aligned}
\lim _{N \rightarrow \infty} \frac{1}{N^{k}} \sum_{n=1}^{N^{k}} \exp \left(\left(\frac{j_{1}}{m}, \cdots, \frac{j_{k}}{m}\right) \cdot \underline{y}_{n}\right) & =\lim _{N \rightarrow \infty} \frac{1}{N^{k}} \prod_{i=1}^{k}\left(\sum_{n=1}^{N} \exp \left(\frac{j_{i}}{m} x_{n i}\right)\right) \\
& =\prod_{i=1}^{k}\left(\lim _{N \rightarrow \infty} \frac{1}{N} \sum_{n=1}^{N} \exp \left(\frac{j_{i}}{m} x_{n i}\right)\right)=0
\end{aligned}
$$

by taking $k=1$ in Theorem 2.6. By elementary estimates, this limit relation implies

$$
\lim _{N \rightarrow \infty} \frac{1}{N} \sum_{n=1}^{N} \exp \left(\left(\frac{j_{1}}{m}, \cdots, \frac{j_{k}}{m}\right) \cdot \underline{y}_{n}\right)=0,
$$

so that $\underline{Y}$ is u.d. in $Z^{k}$ by Theorem 2.6 and Definition 2.5.

Now assume that $\underline{Y}$ is u.d. in $Z^{k}$. Without loss of generality, we shall prove that $X_{1}$ is u.d. in $Z$. Let $m \geqq 2$ be a rational integer, and let $0<j<m$. Then,

$$
\begin{aligned}
\frac{1}{N} \sum_{n=1}^{N} \exp \left(\frac{j}{m} x_{n 1}\right) & =\left(\frac{1}{N} \sum_{n=1}^{N} \exp \left(\frac{j}{m} x_{n 1}\right)\right) \prod_{i=2}^{k}\left(\frac{1}{N} \sum_{n=1}^{N} \exp \left(\frac{0}{m} x_{n i}\right)\right) \\
& =\frac{1}{N^{k}} \sum_{n=1}^{N^{k}} \exp \left(\left(\frac{j}{m}, 0, \cdots, 0\right) \cdot \underline{y}_{n}\right) .
\end{aligned}
$$

Letting $N \rightarrow \infty$, we have

$$
\lim _{N \rightarrow \infty} \frac{1}{N} \sum_{n=1}^{N} \exp \left(\frac{j}{m} x_{n 1}\right)=0
$$

by Theorem 2.6, and the desired conclusion follows from the Weyl criterion for u.d. in $Z$.

The cube method can be used to find an enumeration (without repetition) of all lattice points in $\boldsymbol{Z}^{k}$ into a u.d. sequence in $\boldsymbol{Z}^{k}$ (or, equivalently, an enumeration, without repetition, of all elements of $O$ into a u.d. sequence in $O$ ).

Definition 3.2. Let each of the sequences $X_{1}, \cdots, X_{k}$ be identical to the sequence $0,1,-1,2,-2, \cdots$. Then a sequence $\underline{R}=\left(\underline{r}_{n}\right), n=$ $1,2, \cdots$, arising from the sequences $X_{1}, \cdots, X_{k}$ by the cube method is called a sequence of all lattice points in $\boldsymbol{Z}^{k}$ enumerated by the cube method. 
THEOREM 3.3. A sequence of all lattice points in $Z^{k}$ enumerated by the cube method is u.d. in $\boldsymbol{Z}^{k}$.

Proof. This follows immediately from Theorem 3.1 and the fact that the sequence $0,1,-1,2,-2, \cdots$ is u.d. in $Z$.

Definition 3.4. A lattice point $\underline{x}=\left(x_{1}, \cdots, x_{k}\right) \in Z^{k}$ is called a positive lattice point if $x_{i} \geqq 1$ for $1 \leqq i \leqq k$.

Definition 3.5. Let each of the sequences $X_{1}, \cdots, X_{k}$ be identical to the sequence $1,2,3, \ldots$ Then a sequence $\underline{R}^{+}=\left(\underline{r}_{n}^{+}\right), n=$ $1,2, \cdots$, arising from the sequences $X_{1}, \cdots, X_{k}$ by the cube method is called a sequence of all positive lattice points in $Z^{k}$ enumerated by the cube method.

THEOREM 3.6. A sequence of all positive lattice points in $\boldsymbol{Z}^{k}$ enumerated by the cube method is u.d. in $\boldsymbol{Z}^{k}$.

Proof. This follows immediately from Theorem 3.1 and the fact that the sequence $1,2,3, \cdots$ is u.d. in $Z$.

A variety of other interesting classes of u.d. sequences in $Z^{k}$ can be found by the cube method. We mention a typical example. As usual, $[t]$ denotes the integral part of a real number $t$.

THEOREM 3.7. Let $\underline{R}^{+}=\left(\underline{r}_{n}^{+}\right), n=1,2, \cdots$, be a sequence of all positive lattice points in $\boldsymbol{Z}^{k}$ enumerated by the cube method, with $\underline{\boldsymbol{r}}_{n}^{+}=\left(x_{n 1}, \cdots, x_{n k}\right)$ for $n \geqq 1$, and suppose $a_{1}, \cdots, a_{k}$ are $k$ real numbers. Then the sequence $\underline{X}=\left(\underline{x}_{n}\right), n=1,2, \cdots$, with $\underline{x}_{n}=\left(\left[a_{1} x_{n 1}\right]\right.$, $\left.\cdots,\left[a_{k} x_{n k}\right]\right)$ for $n \geqq 1$, is u.d. in $\boldsymbol{Z}^{k}$ if and only if each $a_{i}$ is irrational or the reciprocal of a nonzero rational integer.

Proof. For $1 \leqq i \leqq k$, let $X_{i}$ be the sequence $X_{i}=\left(\left[n a_{i}\right]\right)$, $n=1,2, \cdots$. It is obvious that $\underline{X}$ is a sequence arising from the sequences $X_{1}, \cdots, X_{k}$ by the cube method. The result follows then from Theorem 3.1 and a result of Niven [8] (see also [4, p. 308]).

4. The Banach-Buck measure. In this section, we shall define a finitely additive measure on $O$, the ring of all algebraic integers in a fixed algebraic number field $K$. This idea was first used by Buck [1] in his discussion of density of sets of rational integers. Later on, M. and S. Uchiyama [9] applied Buck's idea to the theory of uniform distribution of rational integers. See also [3] and [4, Ch. $5, \S 1]$. 
If $I$ is an integral ideal, then the ring $O$ of all algebraic integers of $K$ is partitioned into cosets of $I$. Let $\mathfrak{B}$ consist of the empty set and of all finite unions of cosets of nonzero ideals of $O$. It is easily seen that $\mathfrak{B}$ is an algebra.

DEFINITION 4.1. The set function $\mu: \mathfrak{B} \mapsto \boldsymbol{R}^{+}$(nonnegative real numbers) is defined by $\mu(\varnothing)=0, \mu(E)=1 / \mathscr{N} I$ if $E \in \mathfrak{B}$ is a coset of the nonzero integral ideal $I$, and $\mu\left(E_{1} \cup E_{2}\right)=\mu\left(E_{1}\right)+\mu\left(E_{2}\right)$ if $E_{1}, E_{2} \in \mathfrak{B}$ with $E_{1} \cap E_{2}=\varnothing$.

One checks in a straightforward manner that $\mu$ is well defined. Obviously, $\mu$ is a finitely additive normed measure on $\mathfrak{B}$. The proof of the following simple characterization of u.d. in $O$ can be left to the reader.

THEOREM 4.2. Let $\mathscr{A}=\left(\alpha_{n}\right), n=1,2, \cdots$, be a sequence of elements of $O$. Then $\mathscr{A}$ is u.d. in $O$ if and only if

$$
\lim _{N \rightarrow \infty} \frac{1}{N} \sum_{n=1}^{N} \chi_{E}\left(\alpha_{n}\right)=\mu(E) \text { for every } E \in \mathfrak{B},
$$

where $\chi_{E}$ denotes the characteristic function of the set $E$.

Let $\mu^{*}$ be the outer measure associated with $\mu$. In detail, we define

$$
\mu^{*}(F)=\inf \{\mu(E): F \subseteq E, E \in \mathfrak{B}\}
$$

for every subset $F$ of $O$. The set functions $\mu^{*}$ and $\mu$ coincide on $\mathfrak{B}$.

DEFINITION 4.3. Let $\overline{\mathfrak{B}}$ be the collection of all subsets $F$ of $O$ such that, for any subset $D$ of $O$, we have

$$
\mu^{*}(D)=\mu^{*}(D \cap F)+\mu^{*}\left(D \cap F^{\prime}\right),
$$

where $F^{\prime}$ is the complement of $F$ with respect to $O$. The elements of $\bar{B}$ are called measurable sets.

From general measure theory, we know that $\overline{\mathfrak{B}}$ is an algebra containing $\mathfrak{B}$ with $\mu^{*}$ a finitely additive normed measure on it. The following statements are equivalent:

(1) $F \in \overline{\mathfrak{B}}$;

(2) $\mu^{*}(F)+\mu^{*}\left(F^{\prime}\right)=1$;

(3) for any $\varepsilon>0$, there exist $E_{1}, E_{2} \in \mathfrak{B}$ such that $E_{2} \subseteq F \subseteq E_{1}$ and $\mu\left(E_{1} \backslash E_{2}\right)<\varepsilon$;

(4) $\mu^{*}(F)=\mu_{*}(F)$, where $\mu_{*}(F)=\sup \{\mu(E): F \supseteqq E, E \in \mathfrak{B}\}$.

For $F \in \bar{B}$, we shall write $\mu(F)$ instead of $\mu^{*}(F)$. The measure 
$\mu$ on $\overline{\mathfrak{B}}$ is called the Banach-Buck measure. The following result shows that we could have included the finite subsets of $O$ in the original algebra $\mathfrak{B}$, as was done in [1].

THEOREM 4.4. If $F$ is a finite subset of $O$, then $F \in \bar{B}$ and $\mu(F)=0$.

Proof. Without loss of generality, we may assume that $F$ consists of one algebraic integer only, say $F=\{\alpha\}$. Let $I=m O$, where $m$ is a positive rational integer. Then $\mathscr{N} I=m^{k}$. Obviously, $F \subset \alpha+I$, and so $\mu^{*}(F) \leqq \mu(\alpha+I)=m^{-k}$. Letting $m \rightarrow \infty$, we obtain $\mu^{*}(F)=0$, hence $\mu_{*}(F)=\mu^{*}(F)=0$.

THEOREM 4.5. A set $A \subseteq O$ is of outer measure 1 if and only if $A$ intersects every coset of every nonzero integral ideal.

Proof. Suppose there is a coset $E$ of a nonzero integral ideal such that $A \cap E=\varnothing$. Then $A \subseteq E^{\prime}$, and so $\mu^{*}(A) \leqq \mu\left(E^{\prime}\right)=1-$ $\mu(E)<1$. Now assume that $\mu^{*}(A)<1$. Then there is a set $E \in \mathfrak{B}$ such that $A \subseteq E$ and $\mu(E)<1$. Let $I$ be a nonzero integral ideal such that $E=\bigcup_{i=1}^{s}\left(\alpha_{i}+I\right)$. Since $\mu(E)<1$, there must be a coset $\beta+I$ such that $E \cap(\beta+I)=\varnothing$, and so $A \cap(\beta+I)=\varnothing$.

EXAMPLE 4.6. The set $C$ of all composite algebraic integers of $O$ has outer measure 1. Because of Theorem 4.5, we need only prove that $C \cap(\alpha+I) \neq \varnothing$ for any coset $\alpha+I$ of $I$, where $I$ is an arbitrary nonzero integral ideal. Without loss of generality, we may assume $\alpha \neq 0$. Choose a composite rational integer $m$ satisfying $m \equiv 1(\bmod \mathscr{N} I)$, for instance, $m=(\mathscr{N} I+1)^{2}$. Then $m \alpha \equiv \alpha(\bmod I), m \alpha \neq 0, m \alpha$ is not a prime in $O$ (since $m$ can already be decomposed nontrivially), and $m \alpha$ is not a unit (otherwise, $m$ were a unit). In other words, $m \alpha \in C \cap(\alpha+I)$.

The following two theorems were first observed by M. and S. Uchiyama [9] in the case of rational integers. Later on, Dijksma and Meijer [3] corrected an error in [9]. Our proof is essentially the same as the corrected version of the argument in [9]. For the sake of completeness, we still give the details.

THEOREM 4.7. If $\mathscr{A}=\left(\alpha_{n}\right), n=1,2, \cdots$, is a u.d. sequence in $O$, then

$$
\lim _{N \rightarrow \infty} \frac{1}{N} \sum_{n=1}^{N} \chi_{F}\left(\alpha_{n}\right)=\mu(F) \quad \text { for every } F \in \overline{\mathfrak{B}}
$$


Proof. Let $E \in \mathfrak{B}$ such that $F \subseteq E$. Then,

$$
\frac{1}{N} \sum_{n=1}^{N} \chi_{F}\left(\alpha_{n}\right) \leqq \frac{1}{N} \sum_{n=1}^{N} \chi_{E}\left(\alpha_{n}\right)
$$

for all $N \geqq 1$, and so

$$
\limsup _{N \rightarrow \infty} \frac{1}{N} \sum_{n=1}^{N} \chi_{F}\left(\alpha_{n}\right) \leqq \lim _{N \rightarrow \infty} \frac{1}{N} \sum_{n=1}^{N} \chi_{E}\left(\alpha_{n}\right)=\mu(E)
$$

by Theorem 4.2. It follows that

$$
\limsup _{N \rightarrow \infty} \frac{1}{N} \sum_{n=1}^{N} \chi_{F}\left(\alpha_{n}\right) \leqq \mu^{*}(F) .
$$

Similarly, we can show that

$$
\mu_{*}(F) \leqq \liminf _{N \rightarrow \infty} \frac{1}{N} \sum_{n=1}^{N} \chi_{F}\left(\alpha_{n}\right) .
$$

Since $F \in \overline{\mathfrak{B}}$, we have $\mu_{*}(F)=\mu^{*}(F)=\mu(F)$, and the proof is complete.

TheOREM 4.8. If $\mathscr{A}=\left(\alpha_{n}\right), n=1,2, \cdots$, is a u.d. sequence in $O$, then the set $A$ of elements of $\mathscr{A}$ satisfies $\mu^{*}(A)=1$. Conversely, let $\mathscr{C}=\left(\gamma_{n}\right), n=1,2, \cdots$, be a sequence of all algebraic integers in $O$ enumerated without repetition in such a way that it is u.d. in O. If $\mathscr{A}=\left(\alpha_{n}\right), n=1,2, \cdots$, is a subsequence of $\mathscr{C}$, and if the set $A$ of elements of $\mathscr{A}$ satisfies $A \in \bar{B}$ and $\mu(A)=1$, then $\mathscr{A}$ is u.d. in $O$.

Proof. To prove the first assertion, let $E \in \mathfrak{B}$ such that $A \subseteq E$. Then,

$$
1=\lim _{N \rightarrow \infty} \frac{1}{N} \sum_{n=1}^{N} \chi_{A}\left(\alpha_{n}\right) \leqq \lim _{N \rightarrow \infty} \frac{1}{N} \sum_{n=1}^{N} \chi_{E}\left(\alpha_{n}\right)=\mu(E)
$$

by Theorem 4.2. Therefore, $\mu^{*}(A)=1$.

The second assertion is shown as follows. Denote by $\mathscr{A}(m)$ the number of elements in $\mathscr{A}$ which precede $\gamma_{m}$ in the sequence $\mathscr{C}$ or are equal to $\gamma_{m}$. Then,

$$
\lim _{m \rightarrow \infty} \frac{\mathscr{A}(m)}{m}=\lim _{m \rightarrow \infty} \frac{1}{m} \sum_{n=1}^{m} \chi_{A}\left(\gamma_{n}\right)=\mu(A)=1
$$

by Theorem 4.7. For given $N \geqq 1$, there exists an $m$ with $\alpha_{N}=\gamma_{m}$. Then, for every $E \in \mathfrak{B}$ we have 


$$
\frac{1}{N} \sum_{n=1}^{N} \chi_{E}\left(\alpha_{n}\right)=\frac{1}{N} \sum_{n=1}^{m} \chi_{E \cap A}\left(\gamma_{n}\right)=\frac{m}{\mathscr{A}(m)} \cdot \frac{1}{m} \sum_{n=1}^{m} \chi_{E \cap A}\left(\gamma_{n}\right)
$$

and so

$$
\lim _{N \rightarrow \infty} \frac{1}{N} \sum_{n=1}^{N} \chi_{E}\left(\alpha_{n}\right)=\lim _{m \rightarrow \infty} \frac{1}{m} \sum_{n=1}^{m} \chi_{E \cap A}\left(\gamma_{n}\right)=\mu(E \cap A) .
$$

But $\mu(E)=\mu(E \cap A)+\mu\left(E \cap A^{\prime}\right)$ and $\mu\left(E \cap A^{\prime}\right) \leqq \mu\left(A^{\prime}\right)=1-\mu(A)=0$, so that $\mu(E \cap A)=\mu(E)$. Thus,

$$
\lim _{N \rightarrow \infty} \frac{1}{N} \sum_{n=1}^{N} \chi_{E}\left(\alpha_{n}\right)=\mu(E),
$$

and $\mathscr{A}$ is u.d. in $O$ by Theorem 4.2 .

REMARK. We can even prove that a set $A \subseteq O$ satisfies $\mu^{*}(A)=1$ if and only if its elements can be arranged into a u.d. sequence in $O$. The sufficiency follows, of course, from Theorem 4.8. In the proof of the necessity, techniques of a different type are involved. The reader is referred to [7].

CoROLlary 4.9. If $\mathscr{C}$ is a sequence of all algebraic integers in $O$ which is u.d. in $O$, and if $\mathscr{A}$ is a subsequence of $\mathscr{C}$ such that the set $A$ of elements of $\mathscr{A}$ is measurable and intersects every coset of every nonzero integral ideal, then $\mathscr{A}$ is u.d. in $O$.

Proof. This is a direct consequence of Theorems 4.5 and 4.8 .

EXAMPLE 4.10. There is a sequence $\mathscr{A}$ satisfying the condition in Corollary 4.9 , but still differing from $\mathscr{C}$ by infinitely many terms. Let $[K: Q]=k \geqq 2$ and let $\mathscr{R}=\left(\rho_{n}\right), n=1,2, \cdots$, be a sequence of all algebraic integers in $O$ enumerated by the cube method with respect to a given integral basis (see Definition 3.2 and the remarks following Theorem 2.4). Let $\mathscr{A}$ be the subsequence consisting of all points of $\mathscr{R}$ except those on the axes. Then, obviously, $\mathscr{A}$ differs from $\mathscr{R}$ by infinitely many terms. We shall show that $\mathscr{A}$ satisfies the conditions in Corollary 4.9.

Obviously, $A \cap(\alpha+m O) \neq \varnothing$ for any nonzero rational integer $m$ and any $\alpha \in O$, so that $A$ intersects any coset of any nonzero integral ideal. To show that $A$ is measurable, consider the principal ideal $m O$ for any $m \geqq 2, m \in Z$. There are $m^{k}-k(m-1)-1$ cosets contained completely in $A$. The measure of their union is given by $\left(m^{k}-k m+k-1\right) / m^{k}$, so that

$$
\mu_{*}(A) \geqq 1-\frac{k}{m^{k-1}}+\frac{k-1}{m^{k}} .
$$


Letting $m \rightarrow \infty$, we get $\mu_{*}(A) \geqq 1$. So, $\mu_{*}(A)=1=\mu^{*}(A)$, and $A$ is measurable.

If $k=1$, one may take for $\mathscr{C}$ the sequence $0,1,-1,2,-2, \cdots$ and for $\mathscr{A}$ the subsequence consisting of all positive or negative composite rational integers.

CoROllaRy 4.11. Let $\mathscr{C}$ be as in Corollary 4.9, and suppose $\mathscr{A}$ is a subsequence of $\mathscr{C}$ that is u.d. in $O$. If $\mathscr{A}^{\prime}$, the complement sequence with respect to $\mathscr{C}$, is also u.d. in $O$, then the set $A$ of elements of $\mathscr{A}$ is not measurable.

Proof. By the first part of Theorem 4.8, we know that $\mu^{*}(A)=1$ and $\mu^{*}\left(A^{\prime}\right)=1$. If $A$ were measurable, we would have

$$
\mu^{*}(A)+\mu^{*}\left(A^{\prime}\right)=1,
$$

a contradiction.

Corollary 4.12. Let $\mathscr{C}$ be as in Corollary 4.9. Suppose $\mathscr{A}$ is a subsequence of $\mathscr{C}$, and let $A$ be the set of elements of $\mathscr{A}$. If $A$ is measurable and $\mu(A)=0$, then $\mathscr{A}^{\prime}$ is u.d. in $O$.

Proof. Since $A$ is measurable, so is $A^{\prime}$. Furthermore, $\mu\left(A^{\prime}\right)=$ $1-\mu(A)=1$. Then, by the second part of Theorem $4.8, \mathscr{A}^{\prime}$ is u.d. in $O$.

5. Density. The following notion of density can be thought of as a special case of Buck's general concept of density in [2]. Suppose $W$ is an integral basis for $K$ over $\boldsymbol{Q}$, and let $\mathscr{R}=\left(\rho_{n}\right)$, $n=1,2, \cdots$, be a sequence of all algebraic integers in $O$ enumerated by the cube method with respect to $W$ (see Definition 3.2 and the remarks following Theorem 2.4).

Definition 5.1. If $A \subseteq O$, we define

$$
B(N, A)=B_{W}(N, A)=\sum_{n=1}^{N} \chi_{A}\left(\rho_{n}\right)
$$

where $\chi_{A}$ is the characteristic function of $A$. If the limit $D_{W}(A)=$ $\lim _{N \rightarrow \infty} B(N, A) / N$ exists, then $D_{W}(A)$ is called the relative density of $A$ with respect to $W$.

REMARKs. ( i) $D_{W}(A)$ is independent of the exact arrangement of elements in $\mathscr{R}$, since 


$$
\lim _{N \rightarrow \infty} \frac{B(N, A)}{N}=\lim _{t \rightarrow \infty} \frac{B\left((2 t+1)^{k}, A\right)}{(2 t+1)^{k}} .
$$

(ii) If $F \in \overline{\mathfrak{B}}$, then $D_{W}(F)$ exists and $D_{W}(F)=\mu(F)$. This follows from Theorem 4.7 and the fact that $\mathscr{R}$ is u.d. in $O$.

(iii) Denote by $\overline{\bar{B}}_{W}$ the set of all subsets $T$ of $O$ for which $D_{W}(T)$ exists. Then we have $\mathfrak{B} \subset \overline{\mathfrak{B}} \subseteq \overline{\overline{\mathfrak{B}}}_{W}$.

(iv) If $T \in \overline{\bar{B}}_{W}$, then $\mu_{*}(T) \leqq D_{W}(T) \leqq \mu^{*}(T)$. To prove this, choose $E \in \mathfrak{B}$ such that $T \subseteq E$. Then we have

$$
D_{W}(T)=\lim _{N \rightarrow \infty} \frac{B(N, T)}{N} \leqq \lim _{N \rightarrow \infty} \frac{B(N, E)}{N}=\mu(E),
$$

and so $D_{W}(T) \leqq \mu^{*}(T)$. Similarly, one proves that $\mu_{*}(T) \leqq D_{W}(T)$.

(v) For any $F \in \overline{\mathfrak{B}}$, the relative density is independent of the integral basis chosen. This is a direct consequence of Remark (ii) and the fact that $\mu(F)$ is independent of the integral basis chosen.

The following questions arise naturally. Let $A \in \overline{\bar{B}}_{W}$; if another integral basis $V$ is chosen, is it necessary that $A \in \overline{\overline{\mathcal{B}}}_{V}$ in general? If it were, is it true that $D_{W}(A)=D_{V}(A)$ ? The first question is still open. Our conjecture is that the answer is negative. As to the second question, the answer turns out to be negative.

EXAMPLE 5.2. Let $k \geqq 2$, and let $W=\left\{\omega_{1}, \cdots, \omega_{k}\right\}$ be a given integral basis. We shall show that for every $\varepsilon>0$ there exists a set $A \subseteq O$ and an integral basis $V$ such that $D_{V}(A)=2^{-k}$, but $D_{W}(A)<\varepsilon$. For a positive rational integer $c$, consider the integral basis $V=\left\{\nu_{1}, \cdots, \nu_{k}\right\}$ with $\nu_{1}=\omega_{1}, \nu_{2}=c \omega_{1}+\omega_{2}$, and $\nu_{i}=\omega_{i}$ for $3 \leqq i \leqq k$. Let $A=\left\{\sum_{i=1}^{k} y_{i} \nu_{i} \in O: y_{1} \geqq 0, \quad y_{i}>0\right.$ for $\left.2 \leqq i \leqq k\right\}$. Then we have, of course, $D_{V}(A)=2^{-k}$. Furthermore, we get $B_{W}\left((2 t+1)^{k}, A\right)=t^{k-2} \sum_{j=1}^{t}[j / c]$ for every rational integer $t \geqq 1$. By trivial estimates, we obtain

$$
t^{k-2}\left(\frac{t(t+1)}{2 c}-t\right)<B_{W}\left((2 t+1)^{k}, A\right) \leqq t^{k-2} \frac{t(t+1)}{2 c},
$$

so that

$$
D_{W}(A)=\lim _{t \rightarrow \infty} \frac{B_{W}\left((2 t+1)^{k}, A\right)}{(2 t+1)^{k}}=\frac{1}{2^{k+1} c} .
$$

This can be made smaller than a given $\varepsilon>0$ by choosing $c$ sufficiently large.

The following theorem is concerned with the relationship between relative densities with respect to different integral bases.

Theorem 5.3. Let $W=\left\{\omega_{1}, \cdots, \omega_{k}\right\}$ and $V=\left\{\nu_{1}, \cdots, \nu_{k}\right\}$ be two 
integral bases. Then there exist two positive constants $c_{1}$ and $c_{2}$,

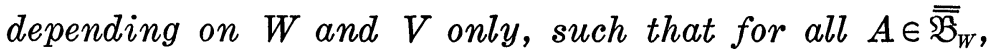

$$
c_{1} D_{W}(A) \leqq \liminf _{N \rightarrow \infty} \frac{B_{V}(N, A)}{N} \leqq \limsup _{N \rightarrow \infty} \frac{B_{V}(N, A)}{N} \leqq c_{2} D_{W}(A) .
$$

Proof. Let $t$ be the smallest positive integer such that the set $\left\{\sum_{i=1}^{k} x_{i} \omega_{i}: x_{i} \in Z,\left|x_{i}\right| \leqq t\right.$ for $\left.1 \leqq i \leqq k\right\}$ contains the set

$$
\left\{\sum_{i=1}^{k} y_{i} \nu_{i}: y_{i} \in Z,\left|y_{i}\right| \leqq 1 \text { for } 1 \leqq i \leqq k\right\} \text {. }
$$

Then for every $N \geqq 1$, the set $\left\{\sum_{i=1}^{k} x_{i} \omega_{i}: x_{i} \in Z,\left|x_{i}\right| \leqq N t\right.$ for $1 \leqq$ $i \leqq k\}$ contains the set $\left\{\sum_{i=1}^{k} y_{i} \nu_{i}: y_{i} \in Z,\left|y_{i}\right| \leqq N\right.$ for $\left.1 \leqq i \leqq k\right\}$. It follows that

$$
B_{V}\left((2 N+1)^{k}, A\right) \leqq B_{W}\left((2 N t+1)^{k}, A\right),
$$

and so

$$
\frac{B_{V}\left((2 N+1)^{k}, A\right)}{(2 N+1)^{k}} \leqq \frac{(2 N t+1)^{k}}{(2 N+1)^{k}} \cdot \frac{B_{W}\left((2 N t+1)^{k}, A\right)}{(2 N t+1)^{k}} .
$$

Therefore,

$$
\limsup _{N \rightarrow \infty} \frac{B_{V}\left((2 N+1)^{k}, A\right)}{(2 N+1)^{k}} \leqq t^{k} D_{W}(A) .
$$

The right-hand side of the inequality follows with $c_{2}=t^{k}$.

The left-hand side can be proved in a similar way. We choose the smallest positive integer $s$ such that the set $\left\{\sum_{i=1}^{k} y_{i} \nu_{i}: y_{i} \in Z\right.$, $\left|y_{i}\right| \leqq s$ for $\left.1 \leqq i \leqq k\right\}$ contains the set $\left\{\sum_{i=1}^{k} x_{i} \omega_{i}: x_{i} \in Z,\left|x_{i}\right| \leqq 1\right.$ for $1 \leqq i \leqq k\}$. Then for every $N \geqq 1$, the set $\left\{\sum_{i=1}^{k} y_{i} \nu_{i}: y_{i} \in Z,\left|y_{i}\right| \leqq N s\right.$ for $1 \leqq i \leqq k\}$ contains the set $\left\{\sum_{i=1}^{k} x_{i} \omega_{i}: x_{i} \in Z,\left|x_{i}\right| \leqq N\right.$ for $1 \leqq$ $i \leqq k\}$. It follows that

$$
B_{W}\left((2 N+1)^{k}, A\right) \leqq B_{V}\left((2 N s+1)^{k}, A\right),
$$

and so

$$
\frac{1}{s^{k}} D_{W}(A) \leqq \liminf _{N \rightarrow \infty} \frac{B_{V}\left((2 N s+1)^{k}, A\right)}{(2 N s+1)^{k}} .
$$

Thus we have the desired result with $c_{1}=s^{-k}$.

REMARK. The values of $t$ and $s$ in the proof of the preceding theorem can be determined as follows. Since $W$ is an integral basis, we have

$$
\nu_{i}=\sum_{j=1}^{k} a_{i j} \omega_{j} \text { for } 1 \leqq i \leqq k \text {, with } a_{i j} \in \boldsymbol{Z}
$$


Every "vertex" of the "unit cube" $\left\{\sum_{i=1}^{k} y_{i} \nu_{i}: y_{i} \in Z,\left|y_{i}\right| \leqq 1\right.$ for $1 \leqq$ $i \leqq k\}$ is of the form $\sum_{i=1}^{k} \varepsilon_{i} \nu_{i}$, where $\varepsilon_{i}=1$ or -1 . However,

$$
\sum_{i=1}^{k} \varepsilon_{i} \nu_{i}=\sum_{j=1}^{k}\left(\sum_{i=1}^{k} \varepsilon_{i} a_{i j}\right) \omega_{j} .
$$

Therefore, $t=\max _{j=1, \ldots, k} \sum_{i=1}^{k}\left|a_{i j}\right| . \quad$ By interchanging the roles of $W$ and $V$, the value of $s$ can be found in the same way.

Corollary 5.4. If $D_{W}(A)=0$ or 1 , then $D_{V}(A)=0$ or 1 , respectively.

Proof. If $D_{W}(A)=0$, then $D_{V}(A)=0$ by Theorem 5.3. If $D_{W}(A)=1$, then $D_{W}\left(A^{\prime}\right)=0$, and so $D_{V}\left(A^{\prime}\right)=0$, which implies $D_{V}(A)=1$.

If the relative density of a given set $A \subseteq O$ is independent of the integral basis chosen, then we simply call it the density of $A$ and denote it by $D(A)$. Let $\overline{\bar{B}}$ be the collection of all $A \cong O$ such that $D(A)$ exists.

We have shown that $\overline{\mathfrak{B}} \cong \overline{\bar{B}}$ and, if $A \cong O$ with relative density 0 or 1 , then $A \in \overline{\overline{\mathfrak{B}}}$. The following question arises: is it necessary, in general, that a set of density 0 or 1 be a measurable set? We shall answer this question by giving the following example.

ExAmpLE 5.5. There exists a set $A \subseteq O$ with $D(A)=0$ such that $A$ is not measurable. Enumerate all distinct cosets of all nonzero ideals of $O$ into a denumerable sequence $\left(E_{n}\right), n=1,2, \ldots$. Let $\mathscr{R}=\left(\rho_{n}\right), n=1,2, \cdots$, be a sequence of all algebraic integers in $O$ enumerated by the cube method with respect to an integral basis $W$. We shall use the notation $\alpha \geqq \beta$ if $\alpha$ is equal to or after $\beta$ in the sequence $\mathscr{R}$. Let $A=\left\{\alpha_{1}, \alpha_{2}, \cdots\right\}$ be constructed inductively as follows: choose $\alpha_{1} \in E_{1}$; then choose $\alpha_{2} \in E_{2}$ with $\alpha_{2} \neq \alpha_{1}$ and $\alpha_{2} \geqq \rho_{4}$; in general, choose $\alpha_{n} \in E_{n}$ with $\alpha_{n} \neq \alpha_{i}$ for $i<n$ and $\alpha_{n} \geqq \rho_{n^{2}}$. This construction is possible since each $E_{n}$ is infinite. Note that $B_{W}\left(N^{2}, A\right) \leqq N$ for $N \geqq 1$, so that $D(A)=0$. We claim that $A$ is not measurable. Indeed, it follows from the above construction that $A$ intersects every coset of every nonzero integral ideal, and so $\mu^{*}(A)=1$ by Theorem 4.5. On the other hand, $\mu_{*}(A) \leqq D(A)=0$ by Remark (iv) following Definition 5.1, hence $A$ is not measurable.

The following question is also of interest: let $A \in \overline{\bar{B}}, D(A) \neq 0,1$; is $A \in \overline{\mathcal{B}}$ in general? The answer turns out to be negative.

EXAMPLE 5.6. For a fixed integral basis $W=\left\{\omega_{1}, \cdots, \omega_{k}\right\}$, consider 
the set $A=\left\{\sum_{i=1}^{k} x_{i} \omega_{i} \in O: x_{1}>0\right\}$. Let $V=\left\{\nu_{1}, \cdots, \nu_{k}\right\}$ be any integral basis. Then, for $N \geqq 1$ we have

$$
(2 N+1)^{k}=2 B_{V}\left((2 N+1)^{k}, A\right)+M(N),
$$

where $M(N)$ is the number of $\alpha=\sum_{i=1}^{k} y_{i} \nu_{i}$ with $\left|y_{i}\right| \leqq N$ for $1 \leqq i \leqq k$, such that the coefficient of $\omega_{1}$ in the representation $\alpha=\sum_{i=1}^{k} x_{i} \omega_{i}$ is zero. Now

$$
\nu_{i}=\sum_{j=1}^{k} a_{i j} \omega_{j} \text { for } 1 \leqq i \leqq k, a_{i j} \in \boldsymbol{Z},
$$

so that $M(N)=\operatorname{card}\left\{\left(y_{1}, \cdots, y_{k}\right) \in Z^{k}: \sum_{i=1}^{k} a_{i 1} y_{i}=0,\left|y_{i}\right| \leqq N\right.$ for $1 \leqq i \leqq k\}$. But at least one of the $a_{i 1}$ is nonzero, so that a trivial upper bound for $M(N)$ is $(2 N+1)^{k-1}$. It follows that

$$
\frac{(2 N+1)^{k}-(2 N+1)^{k-1}}{2} \leqq B_{V}\left((2 N+1)^{k}, A\right) \leqq \frac{(2 N+1)^{k}}{2},
$$

and so $D_{V}(A)=1 / 2$. Since $V$ was arbitrary, we have $D(A)=1 / 2$.

However, $A$ is not measurable. To see this, we note first that $A$ intersects every coset of every nonzero integral ideal, so that $\mu^{*}(A)=1$ by Theorem 4.5. On the other hand, $A$ contains no coset completely, and so $\mu_{*}(A)=0$.

To sum up, we have established the following chain of inclusions:

$$
\mathfrak{B} \subset \overline{\mathfrak{B}} \subset \overline{\mathfrak{B}} \cong \overline{\mathfrak{B}}_{W},
$$

where $W$ is any integral basis. The last inclusion is proper for $k>1$ (see Example 5.2), the other inclusions are proper in all cases (see Theorem 4.4 and Example 5.5). One should observe that $\overline{\overline{\mathfrak{B}}}$ is not an algebra. This is well-known for $k=1$ (see [1]). For $k>1$, we have constructed in Example 5.2 a finite intersection of "halfspaces" (with respect to the integral basis $V$ ) that is not in $\overline{\overline{\mathfrak{B}}}$. However, each individual half-space is in $\overline{\overline{\mathfrak{B}}}$ by the argument in Example 5.6.

We discuss now some relations between density and uniform distribution. We remark that relations of a different type between density of sets of lattice points and uniform distribution mod 1 have been found by Volkmann [11].

LEMma 5.7. Let $\mathscr{R}=\left(\rho_{n}\right), n=1,2, \cdots$, be a sequence of all algebraic integers in $O$ enumerated by the cube method with respect to a given integral basis $W$. Then the subsequence $\mathscr{A}=\left(\alpha_{n}\right)$, $n=1,2, \cdots$, of $\mathscr{R}$ is u.d. in $O$ if and only if 


$$
\lim _{N \rightarrow \infty} \frac{B_{W}(N, A \cap E)}{B_{W}(N, A)}=\mu(E) \text { for every } E \in \mathfrak{B},
$$

where $A$ is the set of elements of $\mathscr{A}$.

Proof. Suppose the condition is satisfied. For any $\alpha_{m}$, there exists an $N$ such that $\alpha_{m}=\rho_{N}$. So

$$
\lim _{m \rightarrow \infty} \frac{1}{m} \sum_{n=1}^{m} \chi_{E}\left(\alpha_{n}\right)=\lim _{N \rightarrow \infty} \frac{B_{W}(N, A \cap E)}{B_{W}(N, A)}=\mu(E)
$$

for every $E \in \mathfrak{B}$, hence $\mathscr{A}$ is u.d. in $O$ by Theorem 4.2.

For the converse, choose $N \geqq 1$ and let $m=B_{W}(N, A)$. Then $\sum_{n=1}^{m} \chi_{E}\left(\alpha_{n}\right)=B_{W}(N, A \cap E)$ for every $E \in \mathfrak{B}$, and so

$$
\lim _{N \rightarrow \infty} \frac{B_{W}(N, A \cap E)}{B_{W}(N, A)}=\lim _{m \rightarrow \infty} \frac{1}{m} \sum_{n=1}^{m} \chi_{E}\left(\alpha_{n}\right)=\mu(E) .
$$

THEOREM 5.8. Let $\mathscr{R}$ be a sequence of all algebraic integers in $O$ enumerated by the cube method with respect to a given integral basis, and let $\mathscr{A}$ be a subsequence of $\mathscr{R}$. If the set $A$ of elements of $\mathscr{A}$ satisfies $D(A)=1$, then $\mathscr{A}$ is u.d. in $O$.

Proof. Without loss of generality, assume $A \neq O$. Let $E \in \mathfrak{B}$ be arbitrary. Then

$$
\begin{aligned}
\frac{B(N, A \cap E)}{B(N, A)}= & \frac{B(N, E)}{N} \cdot \frac{N}{B(N, A)} \\
& -\frac{B\left(N, A^{\prime} \cap E\right)}{B\left(N, A^{\prime}\right)} \cdot \frac{B\left(N, A^{\prime}\right)}{N} \cdot \frac{N}{B(N, A)}
\end{aligned}
$$

for sufficiently large $N$. Since

$$
\lim _{N \rightarrow \infty} \frac{N}{B(N, A)}=1, \quad \lim _{N \rightarrow \infty} \frac{B\left(N, A^{\prime}\right)}{N}=0,
$$

and $0 \leqq B\left(N, A^{\prime} \cap E\right) / B\left(N, A^{\prime}\right) \leqq 1$, it follows that

$$
\lim _{N \rightarrow \infty} \frac{B(N, A \cap E)}{B(N, A)}=\lim _{N \rightarrow \infty} \frac{B(N, E)}{N}=\mu(E),
$$

and so $\mathscr{A}$ is u.d. in $O$ by Lemma 5.7 .

EXAMPle 5.9. The converse of the above theorem is not true. In fact, there is a subsequence $\mathscr{A}=\left(\alpha_{n}\right), n=1,2, \cdots$, of $\mathscr{R}$, a sequence of all algebraic integers in $O$ enumerated by the cube method with respect to the integral basis $W=\left\{\omega_{1}, \cdots, \omega_{k}\right\}$, such that $\mathscr{A}$ is 
u.d. in $O$ but the set $A$ of elements of $\mathscr{A}$ satisfies $D(A)=0$. Let $\mathscr{R}^{+}=\left(\rho_{n}^{+}\right), n=1,2, \cdots$, be the subsequence of $\mathscr{R}$ consisting of the positive lattice points (see Definition 3.4) in the identification of $\mathscr{R}$ with a sequence of lattice points. Now put $\alpha_{n}=\rho_{n}^{+}+n ! \omega_{1}$ for $n=1,2, \cdots$. To show $D(A)=0$, it suffices to prove $D_{W}(A)=0$. For $N \geqq 1$, let $q$ be the smallest positive integer with $N \leqq q !$. Then $B\left((2 N+1)^{k}, A\right) \leqq B\left((2 q !+1)^{k}, A\right) \leqq q+1$, and so

$$
\frac{B\left((2 N+1)^{k}, A\right)}{(2 N+1)^{k}} \leqq \frac{q+1}{(2(q-1) !+1)^{k}} .
$$

This implies already $D_{W}(A)=0$.

Let $m \geqq 2$ be a rational integer. Then

$$
\alpha_{n}=\rho_{n}^{+}+n ! \omega_{1} \equiv \rho_{n}^{+}(\bmod m O)
$$

for all $n \geqq m$. Thus, apart from finitely many initial terms, the sequence $\mathscr{A}$ is identical $\bmod m O$ with the sequence $\mathscr{R}^{+}$. Since the latter is u.d. in $O$ by Theorem 3.6, $\mathscr{A}$ is u.d. $\bmod m O$. By Theorem 2.4, we are done.

Obviously, the uniform distributivity of $\mathscr{A}$ is not sufficient to guarantee $D(A)=1$. We need a stronger hypothesis.

THEOREM 5.10. Let $\mathscr{R}$ be a sequence of all algebraic integers in $O$ enumerated by the cube method with respect to a given integral basis. Furthermore, assume $\mathscr{A}$ is a subsequence of $\mathscr{R}$ containing a nonempty set $E \in \mathfrak{B}$ and $\mathscr{A}$ is u.d. in $O$. Then the set $A$ of elements of $\mathscr{A}$ satisfies $D(A)=1$.

Proof. We have

$$
\begin{aligned}
\frac{B(N, A)}{N} & =\frac{B(N, A)}{B(N, A \cap E)} \cdot \frac{B(N, A \cap E)}{N} \\
& =\left(\frac{B(N, A \cap E)}{B(N, A)}\right)^{-1} \cdot \frac{B(N, E)}{N}
\end{aligned}
$$

for all sufficiently large $N$, so that $\lim _{N \rightarrow \infty} B(N, A) / N=(\mu(E))^{-1} \mu(E)=1$ by Lemma 5.7 and Remark (ii) following Definition 5.1.

The following theorem was first proved by Niven [8] in the case of rational integers. The proof of its generalization to the case of algebraic integers goes through in exactly the same way, and so it is omitted.

THEOREM 5.11. Let $\mathscr{R}$ be a sequence of all algebraic integers in 
$O$ enumerated by the cube method with respect to an integral basis $W$. Let $\mathscr{A}$ be a subsequence of $\mathscr{R}$ with complement sequence $\mathscr{A}^{\prime}$ with respect to $\mathscr{R}$. If $\lim _{\inf _{N \rightarrow \infty}} B_{W}\left(N, A^{\prime}\right) / N>0$ and $\mathscr{A}$ is u.d. in $O$, then $\mathscr{A}^{\prime}$ is u.d. in $O$.

EXAMPLE 5.12. Let the sequence $\mathscr{R}^{+}$be as in Example 5.9. Then $\mathscr{R}^{+}$is u.d. in $O$ by Theorem 3.6 and has the following additional properties: the set $R^{+}$of elements of $\mathscr{R}^{+}$is not measurable and satisfies $D_{W}\left(R^{+}\right)=2^{-k}$, and the complement sequence $\left(\mathscr{R}^{+}\right)^{\prime}$ is u.d. in $O$ (by Theorem 5.11).

Let $\mathscr{R}$ be a sequence of all algebraic integers in $O$ enumerated by the cube method with respect to an integral basis $W$. Let $A_{1} \subseteq$ $A_{2} \subseteq \cdots$ be an increasing sequence of infinite subsets of $O$ with $A=\bigcup_{i=1}^{\infty} A_{i}$. For each $i \geqq 1$, let $\mathscr{A}_{2}$ be the subsequence of $\mathscr{R}$ made up of the elements of $A_{i}$, and let the sequence $\mathscr{A}$ be constructed from $A$ in the same way. Suppose each $\mathscr{A}_{2}$ is u.d. in $O$. It is easily seen that if $\lim _{i \rightarrow \infty} D_{w}\left(A_{i}\right)=1$, then $\mathscr{A}$ is again u.d. in $O$ (use Theorem 5.8). However, in general, $\mathscr{A}$ need not be u.d. in $O$.

EXAMPLE 5.13. Let $W=\left\{\omega_{1}, \cdots, \omega_{k}\right\}$, and let $m \geqq 2$ be a rational integer. Set

$$
B_{i}=\left\{\sum_{j=1}^{k} x_{j} \omega_{j} \in O:-i \leqq x_{j}<0 \text { for } 1 \leqq j \leqq k\right\} \cap m O
$$

$$
\text { for } i=1,2, \cdots \text {, }
$$

and let $A_{i}=R^{+} \cup B_{i}$ for $i=1,2, \cdots$. It is trivial that $A_{i} \subseteq A_{i+1}$ for $i=1,2, \cdots$, and that $\mathscr{A}_{i}$ is u.d. in $O$ for every $i$. We shall show that $\mathscr{A}$ is not u.d. $\bmod m O$. In fact, for any rational integer $t \geqq 1$, we have $A\left((t m)^{k}+t^{k}, m O, \mathscr{A}\right)=2 t^{k}$, and so

$$
\lim _{t \rightarrow \infty} \frac{A\left((t m)^{k}+t^{k}, m O, \mathscr{A}\right)}{(t m)^{k}+t^{k}}=\frac{2}{m^{k}+1} \neq \frac{1}{m^{k}} .
$$

\section{REFERENCES}

1. R. C. Buck, The measure theoretic approach to density, Amer. J. Math., 68 (1946), 560-580.

2. — Generalized asymptotic density, Amer. J. Math., 75 (1953), 335-346.

3. A. Dijksma and H. G. Meijer, Note on uniformly distributed sequences of integers, Nieuw Arch. voor Wisk., (3) 17 (1969), 210-213.

4. L. Kuipers and H. Niederreiter, Uniform Distribution of Sequences, Interscience Tracts, John Wiley and Sons, New York, 1974.

5. L. Kuipers, H. Niederreiter and J.-S. Shiue, Uniform distribution of sequences in the ring of Gaussian integers, Bull. Inst. Math. Acad. Sinica, to appear.

6. H. Niederreiter, On a class of sequences of lattice points, J. Number Theory, 4 
(1972), 477-502.

7. H. Niederreiter and S. K. Lo, Uniform distribution of sequences of algebraic integers, Math. J. Okayama Univ., to appear.

8. I. Niven, Uniform distribution of sequences of integers, Trans. Amer. Math. Soc., 98 (1961), 52-61.

9. M. and S. Uchiyama, A characterization of uniformly distributed sequences of integers, J. Fac. Sci. Hokkaidô Univ., Ser. I, 16 (1962), 238-248.

10. S. Uchiyama, On the uniform distribution of sequences of integers, Proc. Japan Acad., 37 (1961), 605-609.

11. B. Volkmann, On uniform distribution and the density of sum sets, Proc. Amer. Math. Soc., 8 (1957), 130-136.

Received September 6, 1974. The research of the second author was supported by National Science Foundation grant GP-36418X1.

Benedict College

AND

UNIVERSity of CALIFornia Los ANGELES 


\section{PACIFIC JOURNAL OF MATHEMATICS}

\section{EDITORS}

RICHARD ARENS (Managing Editor) University of California

Los Angeles, California 90024

\section{R. A. BEAUMONT}

University of Washington Seattle, Washington 98105
J. DugundjI Department of Mathematics University of Southern Californı Los Angeles, California 90007

D. Gilbarg AND J. Milgram Stanford University Stanford, California 94305

\section{ASSOCIATE EDITORS}

E. F. BECKENBACH

B. H. NeUMANN

F. WOLF

K. YosHidA

\section{SUPPORTING INSTITUTIONS}

UNIVERSITY OF BRITISH COLUMBIA CALIFORNIA INSTITUTE OF TECHNOLOGY UNIVERSITY OF CALIFORNIA MONTANA STATE UNIVERSITY UNIVERSITY OF NEVADA NEW MEXICO STATE UNIVERSITY OREGON STATE UNIVERSITY UNIVERSITY OF OREGON OSAKA UNIVERSITY

\author{
UNIVERSITY OF SOUTHERN CALIFORNIA \\ STANFORD UNIVERSITY \\ UNIVERSITY OF TOKYO \\ UNIVERSITY OF UTAH \\ WASHINGTON STATE UNIVERSITY \\ UNIVERSITY OF WASHINGTON \\ AMERICAN MATHEMATICAL SOCIETY \\ NAVAL WEAPONS CENTER
}




\section{Pacific Journal of Mathematics}

\section{Vol. 61, No. $1 \quad$ November, 1975}

Jiří Adámek, V. Koubek and Věra Trnková, Sums of Boolean spaces represent every

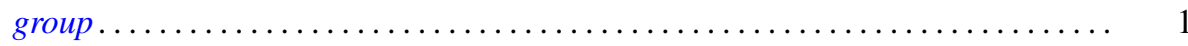

Richard Neal Ball, Full convex l-subgroups and the existence of $a^{*}$-closures of

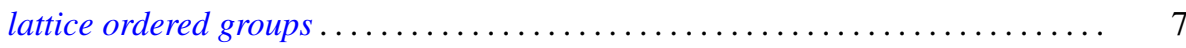

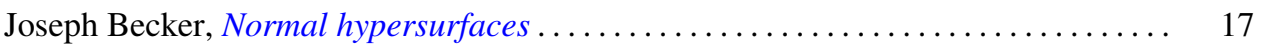

Gerald A. Beer, Starshaped sets and the Hausdorff metric . . . . . . . . . . . . . 21

Dennis Dale Berkey and Alan Cecil Lazer, Linear differential systems with

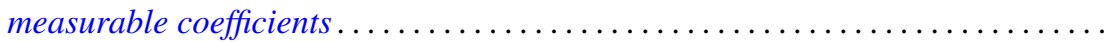

Harald Boehme, Glättungen von Abbildungen 3-dimensionaler

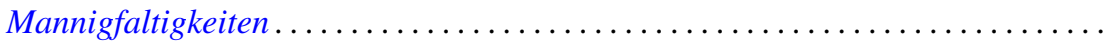

Stephen LaVern Campbell, Linear operators for which $T^{*} T$ and $T+T^{*}$

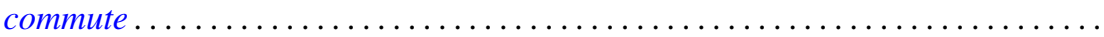

H. P. Dikshit and Arun Kumar, Absolute summability of Fourier series with

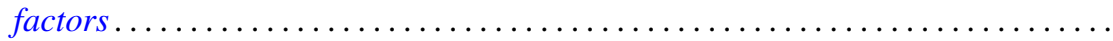

Andrew George Earnest and John Sollion Hsia, Spinor norms of local integral

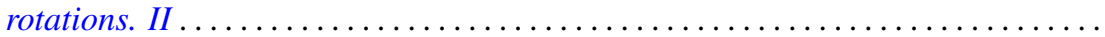

Erik Maurice Ellentuck, Semigroups, Horn sentences and isolic structures .........

Ingrid Fotino, Generalized convolution ring of arithmetic functions . . . . . . . . . . .

Michael Randy Gabel, Lower bounds on the stable range of polynomial rings .......

Fergus John Gaines, Kato-Taussky-Wielandt commutator relations and

characteristic curves

Theodore William Gamelin, The polynomial hulls of certain subsets of $C^{2}$

R. J. Gazik and Darrell Conley Kent, Coarse uniform convergence spaces. . .

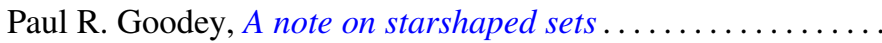

Eloise A. Hamann, On power-invariance

M. Jayachandran and M. Rajagopalan, Scattered compactification for $N \cup\{P\}$. . .

V. Karunakaran, Certain classes of regular univalent functions .

John Cronan Kieffer, A ratio limit theorem for a strongly subadditive set function in a locally compact amenable group .................

Siu Kwong Lo and Harald G. Niederreiter, Banach-Buck measure, density, and uniform distribution in rings of algebraic integers ........

Harold W. Martin, Contractibility of topological spaces onto metric spaces ....

Harold W. Martin, Local connectedness in developable spaces .

A. Meir and John W. Moon, Relations between packing and covering numbers of a tree.

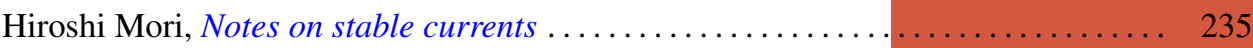

Donald J. Newman and I. J. Schoenberg, Splines and the logarithmic function . . . . 241

M. Ann Piech, Locality of the number of particles operator....

Fred Richman, The constructive theory of $K T$-modules .......

Gerard Sierksma, Carathéodory and Helly-numbers of

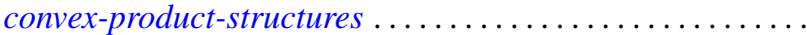

Raymond Earl Smithson, Subcontinuity for multifunctions .... . . 